\title{
Study on Integration of Measurement and Control System for Combine Harvester
}

\author{
Jin Chen ${ }^{1}$, Yuelan Zheng ${ }^{1}$, Yaoming $\mathrm{Li}^{2}$, and Xinhua Wei ${ }^{2}$ \\ ${ }^{1}$ School of Mechanical Engineering, Jiangsu University, \\ Zhenjiang, Jiangsu, 212013, China \\ ${ }^{2}$ Key Laboratory of Modern Agriculture Equipment and Technology, Ministry of Education, \\ Jiangsu University, Zhenjiang, 212013, Jiangsu, China \\ ymli@ujs.edu.cn, yuelan188@163.com, wei_xh@126.com
}

\begin{abstract}
Using CAN (controller area network) field bus and iCAN protocol, an integrated measuring and controlling system for combine harvester is constructed in this paper. ARM is used as the processor of host computer. Lower computers are several measuring and controlling modules of combine harvester. Host computer communicates with lower computers through CAN bus. Information between measurement and control modules can be shared so that combine harvester's whole performance can be improved. LCD on host computer shows working state and values of relative parameters of each module in real-time. Experiment shows that the system can work correctly and reliably.
\end{abstract}

Keywords: Combine harvester, ARM, CAN bus, iCAN protocol, integration.

\section{Introduction}

With supervision and automatic alarm installed in the combine harvester, it is much easier for operator to know the working condition of some running parts and adjust machine's operating parameters in time. So the fault frequency of combine harvester can be reduced, MTBF (the Mean Time Between Failure) can be longer, and the purpose of improving the whole machine's work efficiency can be realized [2]. But at present, the monitoring and controlling objects of combine harvester are mainly walking speed, threshing cylinder speed, state of hydraulic system, etc. Each parameter is basically handled separately. Connections between one another aren't so close [3], and the information can't be shared [4]. Therefore subsystem's function will be influenced and lead to the degradation of the whole machine's performance. To solve this problem, the integration technology is studied in this paper, taking CAN bus as tie. The system is based on the relatively independent modules designed by our studying team. They are Load Feedback Control Module, Real-time Loss Quantity Detecting Module, Electric System Self-checking Module, Mechanical System Fault Monitoring Module, and Hydraulic System Fault Monitoring Module. The system is based on the structure of working process intelligent monitoring and controlling for combine harvester [1], aiming to realize information sharing and improve coordinative relations between parameters effectively. 


\section{The System's General Structure}

The general structure of intelligent monitoring and controlling system for combine harvester is shown in Fig.1 [1]. The system consists of host computer and five measurement and control modules as lower computers. Lower computers realize the function of measuring and controlling of subsystems. Host computer is used for parameter setting, communication and display, etc.

Lower computer modules and their functions are as follows:

1) Load Feedback Control Module: Control the walking speed of combine harvester in real time according to parameters like feed rate, threshing cylinder speed and so on.

2) Real-time Loss Quantity Detecting Module: Detect cleaning loss and entrainment loss real-timely.

3) Electric System Self-checking Module: Detect working status of electric components like battery and generator.

4) Mechanical System Fault Monitoring Module: Offer the early warning and fault alarm for mechanical conveying appliance's jam fault based on parameters like threshing cylinder speed, crop screw conveyor speed, etc.

5) Hydraulic System Fault Monitoring Module: Detect parameters like oil temperature of cooler inlet and outlet, HST forward pressure and recession pressure in real time.

Functions of host computer system and each module of it are as follows:

1) ARM: The processor of host computer.

2) LCD: Display data delivered from lower computers properly.

3) Keyboard: Input parameters.

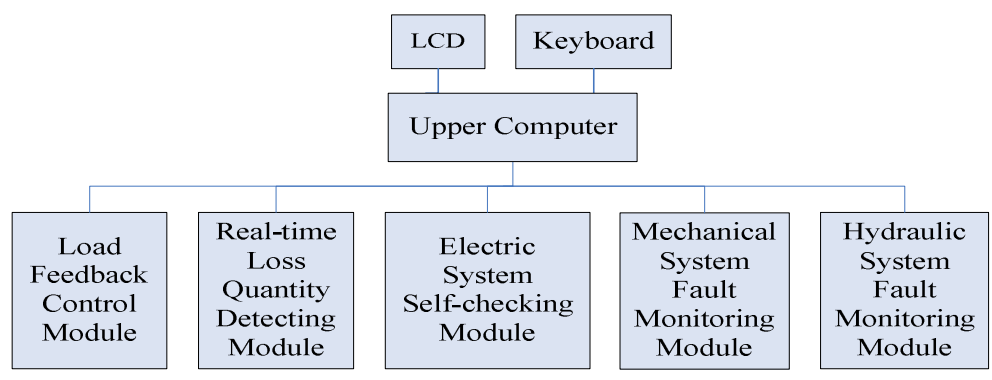

Fig. 1. System's general structure

\section{The System's Realization}

\subsection{ICAN Protocol}

CAN bus, developed in 1983 by Bosch Company of Germany for application in cars, is a serial communication network which can support distributed control and real-time control effectively. CAN bus is of extremely high reliability, high data transmission 
rate, long transmission distance and good real-time performance. It is especially suitable for computer control system in cars, interconnection of industrial field monitoring devices under circumstances of harsh temperature, strong electromagnetic radiation and large vibration [5]. Considering combine harvester's harsh working conditions and its own characteristics, CAN field bus is used in this system.

ICAN is the abbreviation of "Industry CAN-bus Application Protocol", one of the latest application layer protocols of CAN-bus. It is easy to understand, realize, and has properties of real-time and reliability. ICAN protocol uses the way of connection and management that basically similar to CANopen and DeviceNet protocol. The complicated handshake management and resource allocation etc are deleted. Nods on CAN bus is managed in the way of predefined data combination. ICAN protocol's meeting surface is wide, application mode is flexible. It is particularly suitable for distributed data control network under different industrial environment conditions.

Only the extended frame format of CAN message is used in iCAN protocol. It regulates 29 bytes ID and data part of CAN message. ICAN protocol identifier is allocated as shown in Table 1. Table 2 shows how the data part of frame is allocated [6].

Table 1. ICAN message ID allocation

\begin{tabular}{|c|c|c|c|c|c|c|c|c|c|c|}
\hline \multirow{2}{*}{$\begin{array}{l}\text { Frame } \\
\text { ID }\end{array}$} & ID28 & ID27 & $\begin{array}{l}\text { ID26 } \\
\text { ID21 }\end{array}$ & ID20 & ID19 & $\begin{array}{c}\text { ID18 } \\
\text { ID13 }\end{array}$ & ID12 & $\begin{array}{l}\text { ID11 } \\
\text { ID8 }\end{array}$ & $\begin{array}{l}\text { ID7 } \\
\sim \text { ID0 }\end{array}$ & RTR \\
\hline & 0 & 0 & $\begin{array}{c}\text { Source } \\
\text { node } \\
\text { ID }\end{array}$ & 0 & 0 & $\begin{array}{c}\text { Target } \\
\text { node } \\
\text { ID }\end{array}$ & ACK & $\begin{array}{c}\text { FUNC } \\
\text { ID }\end{array}$ & $\begin{array}{c}\text { Source } \\
\text { ID }\end{array}$ & 0 \\
\hline
\end{tabular}

Table 2. Allocation of data part of iCAN frame

\begin{tabular}{|l|l|}
\hline iCAN frame data byte & Function \\
\hline Byte 0 & Segment flag \\
\hline Byte1(Length Flag, Error ID ) & \multirow{2}{*}{ Parameters relevant to FUNC ID } \\
\hline Byte2 7 & \\
\hline
\end{tabular}

Take cooler inlet oil temperature delivered from Hydraulic System Fault Monitoring Module to host computer as an example:

1) Source node ID: 0x03(Nod number of Hydraulic System Fault Monitoring Module)

2) Target node ID: 0x01(Nod number of host computer)

3) ACK: 1(Response frame, no need to be answered)

4) FUNC IN: 0x01(Write port continuously)

5) Source ID: 0xb4(Use this number to represent cooler inlet oil temperature)

6) DLC: 4(Data length)

7) Byte 0:0(No Segmentation for data in this frame)

8) Byte1 Byte4: oil temperature 


\subsection{System's Software Design}

The software of this system is designed by Embedded Visual C++, running under Windows CE circumstance. The host computer software is an important part of the whole measurement and control system. It is mainly used for realizing man-machine interface, receiving and displaying data, communicating with lower computers via CAN bus, setting parameters and showing working state of lower computers etc. Software is designed in the way of modularization. Main modules consisted in this software are as follows:

1) Main interface module: Get into main interface firstly since the system starts running. Main interface gives three buttons, which are "Self-Check", "Set Parameters" and "Real-Time Display". Click one of these three buttons to get into the corresponding interface.

2) Self-check at the beginning interface module: After getting into self-check at the beginning interface, host computer will broadcast message to lower computers asking for their self-check status of the beginning. Meanwhile Timer 1 is activated.

Host computer will do the process according to the information returned from lower computers: if the self-check state is normal, the edit control that represents this module will have a background color of green and show word of "Normal"; if the self-check state is fault, the background color of this edit control is red and word of "Fault" is showed. When Timer 1 reaches its time-out value and host computer still hasn't received self-check message from a lower computer, this module will be regarded as fault. The button relevant to this module showing words of "Error Information" will be able to be seen which is unseen before. Click this button, error code and information will be displayed. Controls of subsequent interfaces relative to this module are forbidden to use. If all of the self-check states of the five lower computer modules are normal finally, the "Self-Check" button on the main interface will be hidden. Otherwise it will keep showing for checking fault information in later time.

3) Parameter setting interface module: In this interface, operator can modify parameters about hydraulic (such as cooler inlet and outlet oil temperature) and loss (1000-grain weight and correction coefficient). After operator input new parameter values and click "OK", host computer will store them and send them to relevant lower computers. Or else, the previous values will be maintained.

4) Real-time display interface: After getting into real-time interface, host computer will broadcast message to lower computer modules for their real-time state data, including the module's current working status and relevant parameters' real-time values. Timer 2 is activated in the mean time. Since then host computer will broadcast to lower computers for real-time status data in certain intervals repeatedly.

Host computer will show the parameter's real-time value in the relevant edit control after received it. If host computer receives a lower computer module's current working state, it will process it based on information received. Take Mechanical System Fault Monitoring Module as an example: if the current state is normal, then its edit 
control will have a green background color and show characters of "Mechanical System is normal"; if current working status is early warning, the edit control has a background color of yellow and shows characters of "Mechanical System is giving early warning"; the background color of the edit control will be red and characters of "Mechanical System is fault" will be displayed if current working state is fault. When a lower computer module's working state at present is fault, fault code and information can be seen by clicking the edit control that represents this module.

\section{Experiment}

\subsection{Hardware Connection of Indoor Experiment}

ARM on the Embedded Experiment Development System OURS-PXA270-EP produced by Beijing Owles Electronic Technology Limited Company is taken as host computer in laboratory. PXA270-EP is a processor based on INTEL XSCALE PXA270, supporting Windows CE 5.0 operating system. There are LCD, serial ports and keyboard in the experiment box. So it can meet the demand of the experiment. Lower computers are Load Feedback Control Module, Real-time Loss Quantity Detecting Module, Electric System Self-checking Module, Mechanical System Fault Monitoring Module and Hydraulic System Fault Monitoring Module and Hydraulic System Fault Monitoring Module.

CSM100, integrated with microprocessor, CAN-bus controller, CAN-bus transceiver, DC-DC module and high photoelectric isolation, is an embedded module used for converting from UART (serial port) to CAN. It is produced by Guangzhou Zhiyuan Electronic Limited Company. It can be embedded in device with UART port conveniently. Without changing the previous hardware structure, the device can get CAN-bus communication interface, realizing data communication between device with UART and CAN-bus network.

Connect serial port 1 of the experiment box with CSM100, and CSM100 with lower computer measurement and control modules. Then the whole indoor experiment

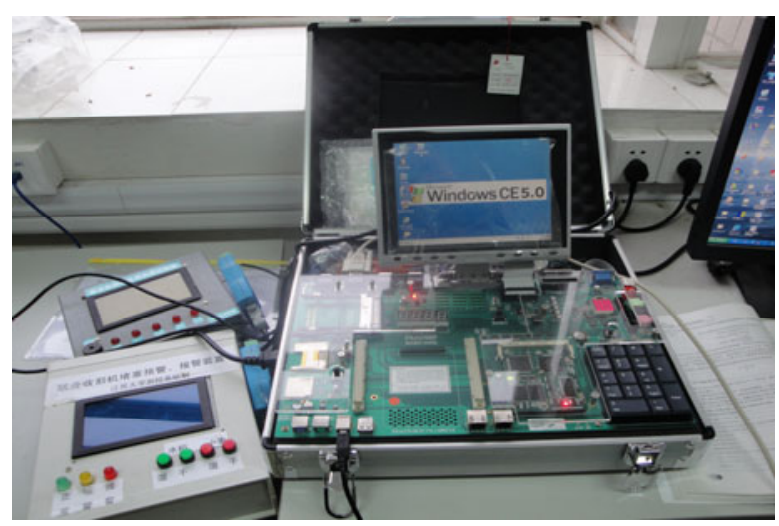

Fig. 2. Devices used in experiment 
system of measurement and control integrated system for combine harvester is constructed, and the indoor communication experiment can be taken. Fig. 2 shows the devices used in the experiment.

\subsection{Indoor Experiment}

Application programs of this system are based on dialog, including the main application program class, serial port class, self-check at the beginning dialog class, hydraulic system parameter setting property page class etc. To avoid main thread being blocked, both reading serial port and writing serial port possess a single thread.

First, use UART debug assistant to make sure that each module of this system, including upper computer and all of the lower computers can respectively communicate correctly. Then do the experiment to test the whole system's performance. The result of the experiment is shown as followed.

Main interface, self-check at the beginning interface, parameter setting interface and real-time display interface are shown in Fig. 3, Fig. 4, Fig. 5, Fig. 6 and Fig. 7 respectively.

Main interface (shown in Fig. 3) is activated when system start running.

Click "Self-Check" button to get into self-check at the beginning interface (shown in Fig. 4) to see self-check states of measurement and control modules and fault information when a module is in fault.

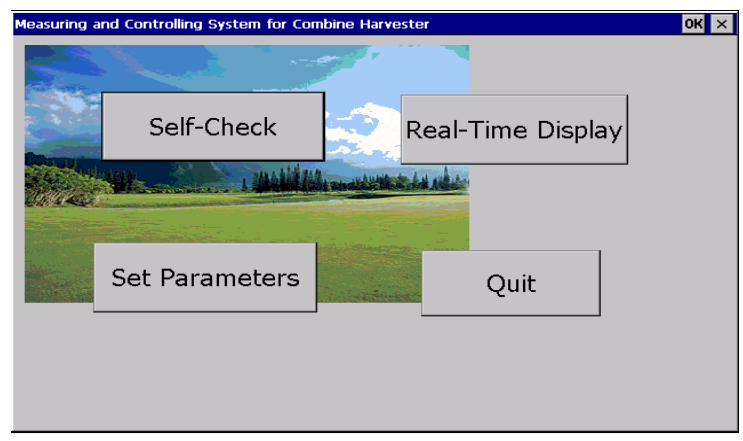

Fig. 3. Main interface

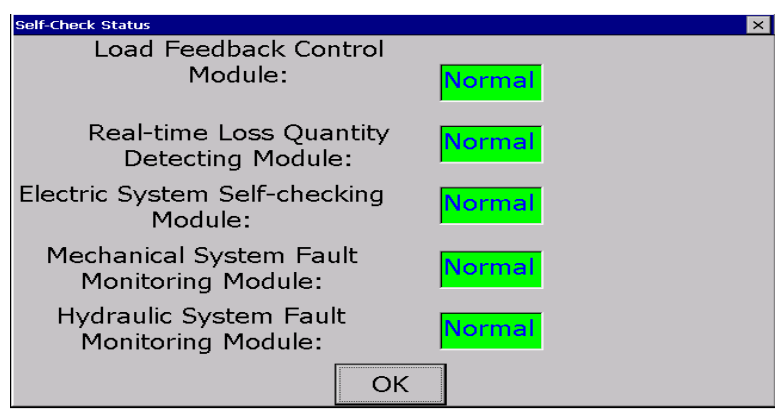

Fig. 4. Self-check at the beginning interface 
Get into parameter setting interface by clicking "Set Parameters" button. Working parameters of hydraulic and loss quantity can be modified here (shown in Fig.5 and Fig.6 respectively).

\begin{tabular}{|c|c|c|c|c|c|}
\hline Set Parameters & & & & & OK $\times$ \\
\hline Set Parameters for Hydraulic System |Set Parar & ameters & 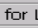 & oss Module | & & \\
\hline $\begin{array}{l}\text { Upper Limit of Cooler Inlet } \\
\text { Oil Temperature: }\end{array}$ & 90.0 & c & $\begin{array}{l}\text { Upper Limit of HST } \\
\text { Fonward Pressure: }\end{array}$ & 10.0 & $\mathrm{MPa}$ \\
\hline $\begin{array}{l}\text { Lower Limit of Cooler Inlet Oil } \\
\text { Temperature: }\end{array}$ & 0.0 & c & $\begin{array}{l}\text { Lower Limit of HST } \\
\text { Fonward Pressure: }\end{array}$ & 1.0 & $\mathrm{MPa}$ \\
\hline $\begin{array}{l}\text { Upper Limit of Cooler Outlet Oil } \\
\text { Temperature: }\end{array}$ & 90.0 & C & $\begin{array}{l}\text { Upper Limit of HST } \\
\text { Recession Pressure: }\end{array}$ & 10.0 & $\mathrm{MPa}$ \\
\hline $\begin{array}{c}\text { Lower Limit of Cooler Outlet Oil } \\
\text { Temperature: }\end{array}$ & 0.0 & C & $\begin{array}{l}\text { Lower Limit of HST } \\
\text { Recession Pressure: }\end{array}$ & 1.0 & $\mathrm{MPa}$ \\
\hline $\begin{array}{l}\text { Upper Limit of Return Oil } \\
\text { Temperature: }\end{array}$ & 90.0 & C & $\begin{array}{l}\text { Upper Limit of Gear } \\
\text { Pump Outlet }\end{array}$ & 10.0 & $\mathrm{MPa}$ \\
\hline $\begin{array}{l}\text { Lower Limit of Return Oil } \\
\text { Temperature: }\end{array}$ & 0.0 & C & $\begin{array}{l}\text { Lower Limit of Gear } \\
\text { Pump Outlet }\end{array}$ & 1.0 & $\mathrm{MPa}$ \\
\hline
\end{tabular}

Fig. 5. Hydraulic parameter setting interface

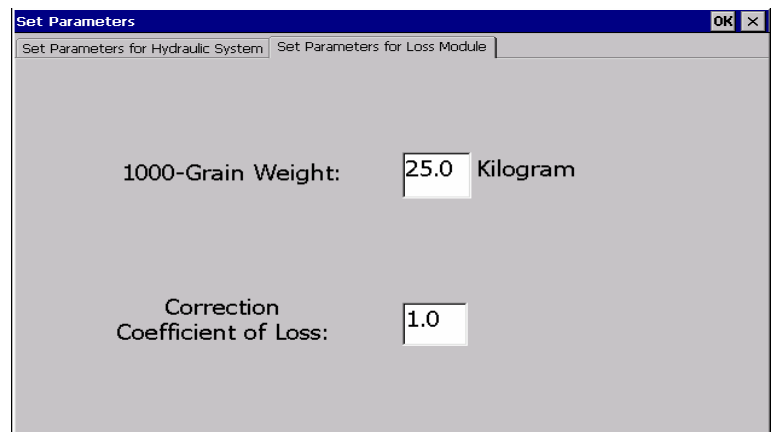

Fig. 6. Loss parameter setting interface

\begin{tabular}{|c|c|c|c|c|c|}
\hline \multicolumn{4}{|l|}{ Real-Time Display } & & $x$ \\
\hline \multicolumn{3}{|c|}{ Load Feedback System is normal } & Crop Screw & 657 & rpm \\
\hline \multicolumn{3}{|c|}{ Loss System is normal } & Oil Temperature & 407 & C \\
\hline \multicolumn{3}{|c|}{ Electric System is normal } & Cooler Inlet: & & \\
\hline \multicolumn{3}{|c|}{ Mechanical System is normal } & of Cooler Outlet: & 1.4 & 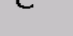 \\
\hline \multicolumn{3}{|c|}{ Hydraulic System is normal } & $\begin{array}{l}\text { Temperature of } \\
\text { Return Oil: }\end{array}$ & 39.9 & C \\
\hline $\begin{array}{l}\text { Walking } \\
\text { Speed: }\end{array}$ & 1.5 & $\mathrm{~m} / \mathrm{s}$ & $\begin{array}{l}\text { HST Forward } \\
\text { Pressure: }\end{array}$ & 8.3 & $\mathrm{MPa}$ \\
\hline $\begin{array}{c}\text { Loss in Real- } \\
\text { Time: }\end{array}$ & 8.61 & $\mathrm{~g} / \mathrm{s}$ & $\begin{array}{l}\text { HST Recession } \\
\text { Pressure: }\end{array}$ & 2.1 & $\mathrm{MPa}$ \\
\hline $\begin{array}{l}\text { Battery } \\
\text { Voltace: }\end{array}$ & 12.2 & $\mathrm{~V}$ & $\begin{array}{c}\text { Oil Temperature } \\
\text { of Gear Pump }\end{array}$ & 4.9 & $\mathrm{MPa}$ \\
\hline $\begin{array}{c}\text { Threshing } \\
\text { Cylinder Speed: }\end{array}$ & 883 & rpm & $\begin{array}{l}\text { Cleanliness of } \\
\text { Hydraulic Oil: }\end{array}$ & Clean & \\
\hline
\end{tabular}

Fig. 7. Real-time display interface 
Click "Real-Time Display" button to get into real-time display interface (shown in Fig. 7). Each lower computer module's current working state and relevant parameters' real-time values are showed here. Click its edit control to see fault information when a module's current working state is fault.

\section{Conclusion}

The experiment shows that this system can realize communication in real-time between host computer and lower computers. LCD on host computer shows working state and parameters of several objects of combine harvester correctly and properly. And the whole machine's performance is increased by shared information between modules. And due to CAN bus's characteristics of high reliability and real-time, the system's reliability is greatly improved. It has a very good application prospect.

Acknowledgments. This work was supported by the Digestion Longitudinal Flow Intelligent Controlling for Rice and Wheat Harvesting Technology \& Equipment Research (2010AA101402) during the Eleventh Five-Year Plan Period.

\section{References}

1. Wei, X.H., Li, Y.M., Chen, J., Song, S.P., Gu, J., Zuo, Z.Y., Ni, J.: System Integration of Working Process Intelligent Monitoring and Controlling Devices for Combine Harvester. Agricultural Engineering Journal 20, 56-60 (2009) (in Chinese)

2. Zhang, S.H., Chen, J.: A Study on the Auto-alarm System of Combine Harvester. Agricultural Mechanization Study 8, 68-73 (2002) (in Chinese)

3. Wang, J.X.: Automotive Electronics Technology Integrated Control System Based on CAN Bus. Harbin Industrial University Journal 5, 811-814 (2006) (in Chinese)

4. Li, J.L., Yu, L., Liu, H., Zhou, H.L.: State-of-Art and Prospect on Combine Monitor System. Modern Agricultural Equipments 12, 46-48 (2005) (in Chinese)

5. Wang, L.M., Xia, L., Shao, Y., Yan, X.L.: The Design and Application of CAN Field Bus System. Electronic Industrial Press, Beijing (2008) (in Chinese)

6. Zhou, L.G.: ICAN Field Bus Principle and Application. Beijing Aeronautics and Astronautics University Press, Beijing (2007) (in Chinese) 